\title{
Cost Effective Profiling of Neodymium-Doped Vanadate Laser Pulses
}

\author{
Raymond Edziah ${ }^{1}$ \\ ${ }^{1}$ Center for Advanced Studies in Photonic Research, University of Maryland, Baltimore County, 1000 Hilltop \\ Circle, Baltimore, Maryland, USA \\ Correspondence: Raymond Edziah, Center for Advanced Studies in Photonic Research, University of Maryland, \\ Baltimore County, 1000 Hilltop Circle, Baltimore, USA. Tel: 1-973-986-3409. E-mail: redziah@ucc.edu
}

Received: January 17, 2018

Accepted: February 25, 2018

Online Published: March 30, 2018

doi:10.5539/apr.v10n2p39

URL: https://doi.org/10.5539/apr.v10n2p39

\begin{abstract}
As environmental conditions and component degradation and failure are known to affect the performance of ultrafast lasers, it is important to monitor their state in any nonlinear optical study. This may be achieved by measuring the temporal width of the laser pulses using an autocorrelator. In this work, an autocorrelator for measuring the pulsewidth of mode-locked lasers was custom-built using pieces of equipment usually found in a typical ultrafast optics laboratory. The assembled equipment was tested using SESAM (Saturable Semiconductor Absorber Mirror) mode-locked neodymium-doped vanadate $\left(\mathrm{Nd}: \mathrm{YVO}_{4}\right)$ laser having manufacturer specified average output power of $1.6 \mathrm{~W}$ and $10 \mathrm{ps}$ pulsewidth. Using the background-free autocorrelation technique, the pulse width of the laser was measured to be $10.4 \mathrm{ps}$. This type of autocorrelator is cost effective and may be handy in situations where research funds are limited; a scenario commonly experienced in research laboratories of developing countries. Additionally, the processes involved in assembling the autocorrelator provide a useful learning experience for new researchers. The study also outlined the processes involved in modifying an existing autocorrelation setup in order to measure laser beam spot size; a useful parameter in nonlinear optical studies.
\end{abstract}

Keywords: autocorrelation, z-scan, second harmonic generation, knife-edge, spot size, semiconductor saturable absorber mirror

\section{Introduction}

Since their invention in 1960 (Maiman, 1960), lasers have been employed in diverse fields such as medicine (Vij \& Mahesh, 2013), optical communication (Marell et al., 2011), optical metrology (Silver et al., 2007), military (Anderberg \& Wolbarsht, 2013) among others. Ultrafast lasers have been particularly helpful in the study of nonlinear optical processes that require high intense laser lights. However, just as any other device, the performance of a laser degrades with time due to factors such as gradual component degradation and environmental conditions such as fluctuating temperature and humidity and high levels of dust. As such, it is important to monitor the performance of a laser on a regular basis.

The most fundamental method of checking the state of a laser is to determine its output power and compare the result with the manufacturer's specified value. On the other hand, a mode-locked laser's performance can appropriately be monitored by estimating its temporal pulse width. However, the tools available for measuring the pulse duration of lasers are limited in scope due to unavailability of appropriate detectors of the required time resolution. Until now, the only detector with a time resolution within the femtosecond and attosecond regimes is the streak camera (Itatani et al., 2002; Frühling et al., 2009; Constant, Taranukhin, Stolow, \& Corkum, 1997; Strickland \& Mourou, 1985) and they are quite expensive. In view of this, subpicosecond and femtosecond laser pulses can be measured by employing the pulses themselves in what is commonly known as intensity autocorrelation, even if the detector itself is much slower. In this technique, the intensity profile of the optical pulse is correlated with itself by mixing two optical pulses from the laser in a nonlinear optical medium to yield sum-frequency generation (in what is known as second harmonic generation, SHG). Although commercial autocorrelators are currently available on the market, they are beyond the reach of many research laboratories especially ultrafast laser users in the developing countries. In this case, custom-built autocorrelators may be preferable. Another advantage of building an autocorrelator from the scratch is that the experience the task provides serves as a valuable laser beam alignment training exercise for new researchers in the laboratory. This work will outline the necessary steps involved in the construction of an autocorrelator in a research laboratory and demonstrate its application in determining the temporal width of a laser pulse. 
One important parameter in any nonlinear optical characterization study is a laser's spot size. Specifically, the beam waist ( $1 / \mathrm{e}^{2}$ of the intensity) of a focused Gaussian laser beam is perhaps one of the most sensitive parameters in z-scan (Sheik-Bahae, Said, Wei, Hagan, \& Van Stryland, 1990) measurements and thermal lens spectroscopy study (Baesso, Shen, Snook, \& Mode, 1994) since a precise knowledge of a focused spot size of a laser is necessary for accurate determination of its intensity. Many different methods have been developed to measure laser beam radius and these include the slit scan (Kiang \& Lang, 1983; Khosrofian \& Garetz, 1983), and pinhole (Bilger \& Habib, 1985) techniques. However, the most versatile of these approaches is the knife-edge method (Cannon, Gardner, \& Cohen, 1980; de Araújo, Silva, de Lima, Pereira, \& de Oliveira, 2009). This is a beam profiling technique that allows for quick, inexpensive, and accurate determination of beam parameters with the possibility to adapt it for a wide range of wavelengths and high power beams. This work will also highlight the steps involved in adapting an existing autocorrelation setup to measure the spot size of a laser beam.

\section{Autocorrelator Construction and Demonstration of its Use}

A schematic representation of the designed and assembled autocorrelator for measuring the temporal width of Time-bandwidth's SESAM (Semiconductor Saturable Absorber Mirror) modelocked laser (wavelength, $\lambda=1064$ $\mathrm{nm}$, average power, $\mathrm{P}_{\mathrm{avg}}=1.64 \mathrm{~W}$, pulsewidth, $\tau_{\mathrm{p}}=10 \mathrm{ps}$ ) is shown in Figure 1 . The major components of the autocorrelator are modified Michelson's interferometer arrangement consisting of two retro-reflectors, beam splitter (BS), second-harmonic generation (SHG) crystal, photodetector (D), focusing lens (L) and mirrors (M).

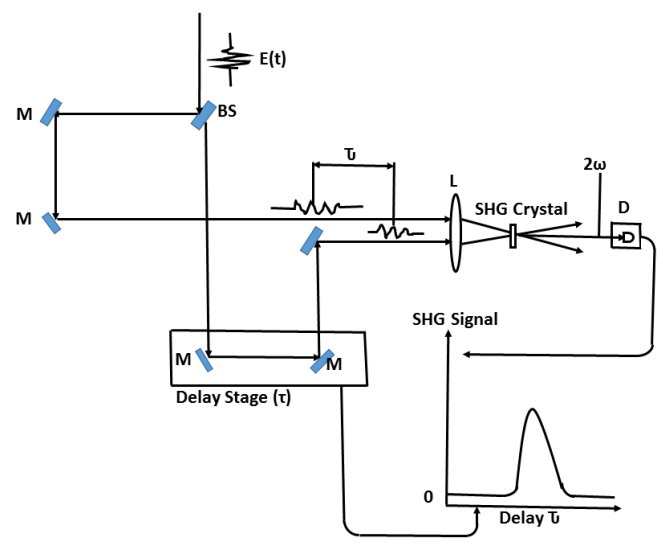

Figure 1. A schematic diagram of assembled autocorrelator

The laser beam is first aligned straight and then divided using a beam splitter. The two noncollinear beams are directed into the two arms of the modified Michelson interferometer. The relative time delay between the two beams is achieved by having one arm of the interferometer fixed and varying the other with the help of steppermotor controlled translation stage (Newport ESP 300 translation stage of total travel range of $250 \mathrm{~mm}$ and resolution step size of $1 \mu \mathrm{m}$ ). The two noncollinear beams are focused into the SHG material, which in this case is a $2 \mathrm{~mm}$-thick Potassium Titanyl Phosphate (KTP) crystal. Upon passage through the crystal, the fundamental beams are blocked by using appropriate beam stops. The second-harmonic signal which is proportional to the product of the intensities of the two beams is detected at an angle bisecting the angle between the two focused fundamental beams. The intrinsic speed of this electronic nonlinearity is on the order of $10^{-15} \mathrm{~s}$ which is utilized in the measurement of the pulsewidth. The second-harmonic signal is produced only when both pulses are present in the crystal, thereby eliminating a background signal, hence the name background-free SHG.

The detection system consists of a silicon photodiode detector whose analog output is filtered by a lock-in amplifier (EG\&G 7260 DSP Lock-in amplifier). One arm of the interferometer is chopped using an appropriate EG\&G light chopper (model 197) which serves as a reference signal for the lock-in amplifier. The output of the lock-in amplifier contains the SHG autocorrelation signal as a function of the relative delay between the two arms of the interferometer. The signal is stored in a computer using LabVIEW enabled National Instruments USB-600 data acquisition card. A computer reads the signal from the lock-in amplifier triggered by the pulses from the stepper motor and plots the signal as a function of the relative time delay as represented schematically in Figure 1. It must be pointed out that the temporal resolution of the measurement is not obtained from the bandwidth of the electronics used, but it is rather dependent on the ability to accurately control the path difference between the two arms of the interferometer and the speed of the electronic nonlinearity responsible for the SHG. 


\section{Evaluation of Temporal Pulse Width}

As the nonlinear optical techniques for laser pulse width measurements do not provide a direct display of the pulse shape but instead give measurements of the correlation functions, the second-order autocorrelation function of the intensity $I(t)$ is expressed as (Ippen \& Shank, 1997):

$$
\begin{aligned}
G^{(2)}(\tau) & =\int_{-\infty}^{+\infty} I^{(\omega)}(t) I^{(\omega)}(t+\tau) d t \\
& =\int_{-\infty}^{+\infty} I^{(\omega)}(t) I^{(\omega)}(t-\tau) d t
\end{aligned}
$$

where $\tau$ is the optical delay between the two fundamental pulses $I^{(\omega)}(t)$ and $I^{(\omega)}(t+\tau)$ oscillating at a frequency $\omega$. This is the function obtained through SHG in the KTP crystal since the SHG intensity $I^{(2 \omega)}$ is proportional to the product of the intensities of the two fundamental pulses. If $I^{(\omega)}(t)$ is a single isolated pulse, then optical autocorrelation function $G^{(2)}(\tau)$ vanishes for large relative delay $(\tau)$ and its half-width provides a measure of the duration of $I^{(\omega)}(t)$.

It must be emphasized that in using the autocorrelation technique for precise determination of a pulsewidth requires further knowledge or assumptions about the shape of $I^{(\omega)}(t)$. In this study, a Gaussian profile was assumed since the Nd: $\mathrm{YVO}_{4}$ laser pulses are generally known to be Gaussian. The intensity autocorrelation function of the Vanadate laser is shown in equation (2) and the assumed pulse shape can be expressed as (Ippen \& Shank, 1997):

$$
I(t)=\exp -\left[\frac{4(\ln 2) t^{2}}{\tau_{p} / 2}\right]
$$

which gives a ratio of $\Delta \tau_{\text {Auto }} / \tau_{p}=1.41$, where $\Delta \tau_{\text {Auto }}$ and $\tau_{p}$ are the autocorrelation full-width at half-

maximum (FWHM) and the actual width of the laser pulse, respectively. Figure 2 shows the autocorrelation data and its theoretical fit from which $\Delta \tau_{\text {Auto }}=14.66 \mathrm{ps}$ and $\tau_{p}=10.4 \mathrm{ps}$.

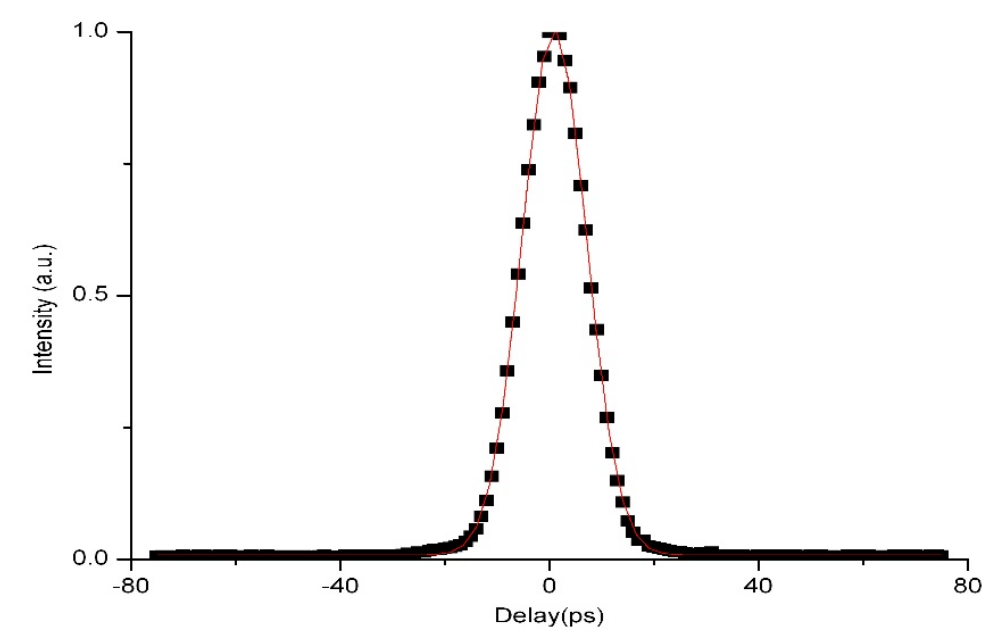

Figure 2. Intensity autocorrelation profile of SESAM (Saturable Semiconductor Absorber Mirror) mode-locked $1064 \mathrm{~nm}$ neodymium-doped vanadate $\left(\mathrm{Nd}: \mathrm{YVO}_{4}\right)$ laser with a fit to a Gaussian temporal pulse shape

\section{Theory and Experiment of Laser Beam Spot Size Measurement}

The study considers a radially symmetric Gaussian laser beam with intensity profile described by

$$
I(x, y)=I_{o} \exp \left[-\frac{\left(x-x_{o}\right)^{2}+\left(y-y_{o}\right)^{2}}{w^{2}}\right]
$$


where $I_{\mathrm{o}}$ is the peak intensity at the centre of the beam located at $\left(x_{0}, y_{0}\right)$ and $x, y$ are the transverse coordinates of any point with respect to an origin conveniently chosen at the beginning of the experiment, and $w$ is the beam radius, measured at a position where the intensity decreases to $1 / e^{2}$ times its maximum value $I_{\mathrm{o}}$. The normalized transmitted power is given by the integral

$$
P_{N}=\frac{\int_{-\infty-\infty}^{x} \int_{-\infty}^{\infty} I\left(x^{\prime}, y\right) d y d x^{\prime}}{\int_{-\infty}^{\infty} \int^{\infty} I\left(x^{\prime}, y\right) d y d x^{\prime}}
$$

such that

$$
P_{N}(x)=\frac{1}{2}\left[1+\operatorname{erf}\left(\frac{x-x_{o}}{w}\right)\right]
$$

where erf is the error function.

A schematic diagram of the experimental setup for measuring the beam waist radius of the $\mathrm{Nd} \mathrm{YVO}_{4}$ laser is represented in Figure 3 (de Araújo, Silva, de Lima, Pereira, \& de Oliveira, 2009).

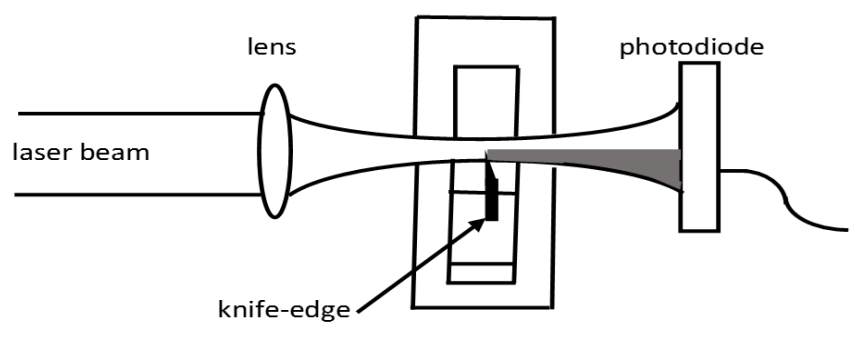

translation stage

Figure 3. A schematic diagram of the knife-edge experimental setup for measuring laser beam waist radius. The shadow caused by the knife-edge is represented by the gray colour

The arrangement utilizes a motorized translation stage onto which a mechanical single axis crossed roller linear translation stage is mounted. The beam is aligned straight and focused using a $10-\mathrm{cm}$ focal length lens and the total power is measured when it is not blocked by the knife-edge. The position of the translation stage is controlled by a LabVIEW interfaced computer and the transmitted power is measured as a function of the transverse position of the knife-edge. Figure 4 shows a plot of the knife-edge data and its theoretical fit based on Gaussian error function theory (Abramowitz \& Stegun, 1964) as represented in equation (5). From the fit, the beam diameter was determined to be $(0.032 \pm 0.002) \mathrm{mm}$.

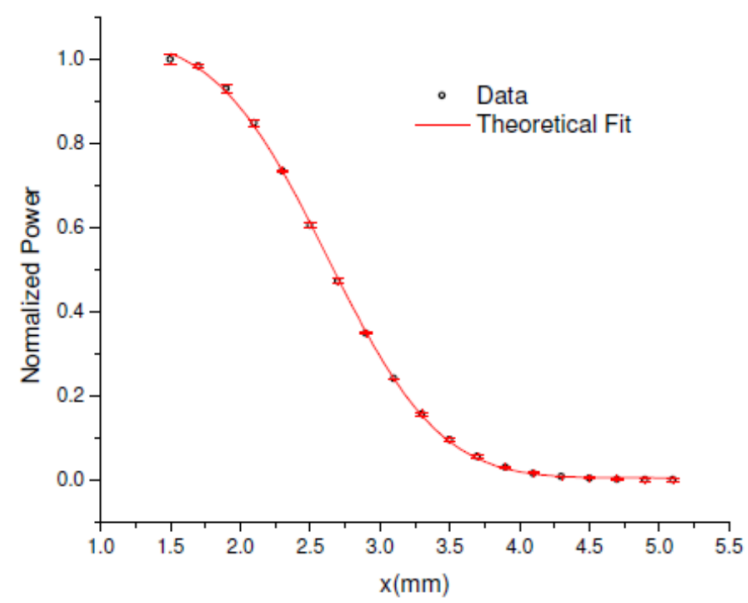

Figure 4. Knife-edge measurement of the beam waist radius $(w)$ of neodymium-doped vanadate $\left(\mathrm{Nd} \mathrm{YVO}_{4}\right)$ laser using a Gaussian error function 


\section{Discussion and Conclusion}

This work outlined the necessary steps involved in the design and construction of a custom-made autocorrelator for measuring the temporal width of an ultrafast laser pulse. The study utilized noncollinear background-free SHG in a 2-mm thick KTP crystal to measure the autocorrelation pulsewidth of the $\mathrm{Nd}: \mathrm{YVO}_{4}$ laser pulses. By choosing an assumed Gaussian pulse shape, the autocorrelation data was theoretically fitted and the actual pulsewidth of the laser determined. This autocorrelator is cost-effective and comes handy in cases where the budget of a modelocked laser user is tight since most of the components needed in assembling it may be readily found in the laboratory. The study also highlighted the process of modifying an existing autocorrelator setup to measure the spot size of a laser beam using the knife-edge approach. This parameter is perhaps one of the most sensitive quantities in nonlinear characterization of materials for optical limiting and switching applications and can readily be determined using the modified autocorrelator arrangement outlined in this study.

\section{References}

Abramowitz, M., \& Stegun, I. A. (1964). Handbook of mathematical functions: with formulas, graphs, and mathematical tables. Courier Corporation.

Anderberg, B., \& Wolbarsht, M. L. (2013). Laser weapons: the dawn of a new military age. Springer.

Baesso, M. L., Shen, J., \& Snook, R. D. (1994). Mode-mismatched thermal lens determination of temperature coefficient of optical path length in soda lime glass at different wavelengths. Journal of applied physics, 75(8), 3732-3737. https://doi.org/10.1063/1.356045

Bilger, H. R., \& Habib, T. (1985). Knife-edge scanning of an astigmatic Gaussian beam. Applied optics, 24(5), 686-690. https://doi.org/10.1063/1.356045

Cannon, B., Gardner, T. S., \& Cohen, D. K. (1986). Measurement of 1- $\mu$ m diam beams. Applied optics, 25(17), 2981-2983. https://doi.org/10.1364/AO.25.002981

Constant, E., Taranukhin, V. D., Stolow, A., \& Corkum, P. B. (1997). Methods for the measurement of the duration of high-harmonic pulses. Physical Review A, 56(5), 3870-3878. https://doi.org/10.1103/PhysRevA.56.3870

de Araújo, M. A., Silva, R., de Lima, E., Pereira, D. P., \& de Oliveira, P. C. (2009). Measurement of Gaussian laser beam radius using the knife-edge technique: improvement on data analysis. Applied optics, 48(2), 393396. https://doi.org/10.1364/AO.48.000393

Frühling, U., Wieland, M., Gensch, M., Gebert, T., Schütte, B., Krikunova, M., ... Plönjes, E. (2009). Single-shot terahertz-field-driven X-ray streak camera. Nature Photonics, 3(9), 523-528. https://doi.org/10.1038/nphoton. 2009.160

Ippen, E. P., \& Shank, C. V. (1977). Techniques for Measurement in Ultrashort Light Pulses. In S. L. Shapiro (Ed.), Topics in Applied Physics. New York: Springer Verlag.

Itatani, J., Quéré, F., Yudin, G. L, Ivanov, M. Y., Krausz, F., \& Corkum, P. B. (2002). Attosecond streak camera. Physical Review Letters, 88(17), 173903. https://doi.org/10.1103/PhysRevLett.88.173903

Khosrofian, J. M., \& Garetz, B. A. (1983). Measurement of a Gaussian laser beam diameter through the direct inversion of knife-edge data. Applied Optics, 22(21), 3406-3410. https://doi.org/10.1364/AO.22.003406

Kiang, Y. C., \& Lang, R. W. (1983). Measuring focused Gaussian beam spot sizes: a practical method. Applied optics, 22(9), 1296-1297. https://doi.org/10.1364/AO.22.001296

Maiman, T. H. (1960). Stimulated optical radiation in ruby. Nature, 187(4736), 493-494. https://doi.org/10.1038/187493a0

Marell, M. J., Smalbrugge, B., Geluk, E. J., van Veldhoven, P. J., Barcones, B., Koopmans, B., ... Hill, M. T. (2011). Plasmonic distributed feedback lasers at telecommunications wavelengths. Optics Express, 19(16), 15109-15118. https://doi.org/10.1364/OE.19.015109

Sheik-Bahae, M., Said, A. A., Wei, T. H., Hagan, D. J., \& Van Stryland, E. W. (1990). Sensitive measurement of optical nonlinearities using a single beam. IEEE journal of quantum electronics, 26(4), 760-769. https://doi.org/10.1109/3.53394

Silver, R. M., Barnes, B. M., Attota, R., Jun, J., Stocker, M., Marx, E., \& Patrick, H. J. (2007). Scatterfield microscopy for extending the limits of image-based optical metrology. Applied optics, 46(20), 4248-4257. https://doi.org/10.1364/AO.46.004248 
Strickland, D., \& Mourou, G. (1985). Compression of amplified chirped optical pulses. Optics communications. 55(6), 447-449. https://doi.org/10.1016/0030-4018(85)90151-8

Vij, D. R., \& Mahesh, K. (Eds.). (2013). Medical applications of lasers. Springer Science \& Business Media.

\section{Copyrights}

Copyright for this article is retained by the author(s), with first publication rights granted to the journal.

This is an open-access article distributed under the terms and conditions of the Creative Commons Attribution license (http://creativecommons.org/licenses/by/4.0/). 